\title{
The Observation Campaign of SS 433 in April 2006
}

\author{
T. Kotani ${ }^{* a}$, K. Kubota ${ }^{b}$, M. Namiki ${ }^{c}$, N. Kawai ${ }^{a}$, Y. Ueda ${ }^{b}$, S. Trushkin ${ }^{d}$, S. Fabrika ${ }^{d}$, \\ V. Afanasiev ${ }^{d}$, P. Abolmasov ${ }^{d}$, K. Kinugasa ${ }^{e}$, T. Nagata $^{b}$, T. Irsmambetova ${ }^{f}$, \\ T. Tsukagoshi ${ }^{g}$, K. Nakanishi $^{h}$, M. Tsuboi ${ }^{h}$, S. Ozaki ${ }^{b i}$, K. Yanagisawa ${ }^{j}$, \\ S. Nishiyama ${ }^{k}$, T. Shimokawabe ${ }^{a}$, Y. Yatsu ${ }^{a}$, T. Ishimura ${ }^{a}$, and K. Fujisawa ${ }^{l}$ \\ ${ }^{a}$ Tokyo Tech, 2-12-1 O-okayama, Meguro, Tokyo 152-8551, Japan \\ ${ }^{b}$ Kyoto University, Kitashirakawa-Oiwake-cho, Sakyo-ku, Kyoto 606-8502, Japan \\ ${ }^{c}$ Osaka University, 1-1 Machikaneyama, Toyonaka, Osaka 560-0043, Japan \\ ${ }^{d}$ Special Astrophysical Observatory, Nizhnij Arkhyz, Karachaevo-Cherkassia 369167, Russia \\ ${ }^{e}$ Gunma Astronomical Observatory, 6860-86 Nakayama, Agatsuma, Gunma 377-0702, Japan \\ ${ }^{f}$ Crimean Astrophysical Observatory, Nauchny, Crimea 98409, Ukraine \\ ${ }^{g}$ SOKENDAI, Minamimaki, Minamisaku, Nagano 384-1305, Japan \\ ${ }^{h}$ Nobeyama Radio Observatory, Minamimaki, Minamisaku, Nagano 384-1305, Japan \\ ${ }^{i}$ Nishi-Harima Astronomical Observatory, Sayo-cho, Hyogo 679-5313, Japan \\ ${ }^{j}$ Okayama Astrophysical Observatory/NAOJ/NINS, 3037-5 Honjo, Okayama 719-0232, Japan \\ ${ }^{k}$ Osaka Kyoiku University, 4-698-1 Asahigaoka, Kaibara, Osaka 582-8582, Japan \\ ${ }^{l}$ Yamaguchi University, 1677-1 Yoshida, Yamaguchi, Yamaguchi 753-8512, Japan \\ E-mail: kotani@hp.phys.titech.ac.jp
}

A radio-IR-optical-X-ray observation campaign of SS 433 has been performed in April 2006, when the jet axis is almost perpendicular to the line of sight. Five flares have been detected during the campaign by radio monitoring observation with RATAN-600. The X-ray astronomical satellite Suzaku observed the source in and out of eclipse. In the X-ray data out of eclipse, the flux shows a significant variation with a time scale of hours. The source seems to be in the active state during the campaign. The observation logs and preliminary results are presented.

VI Microquasar Workshop: Microquasars and Beyond September 18-22 2006

Società del Casino, Como, Italy

\footnotetext{
${ }^{*}$ Speaker.
} 


\section{Introduction}

SS 433 is the unique microquasar known for the very stable continuous jet emanating at a quarter of the speed of light $[14,5]$. The optical and X-ray spectra are abundant in pairs of Dopplershifted emission lines from the bipolar jets. The emission lines are evidence that the jet plasma contains baryons, while other microquasars' jets do not show such Doppler-shifted emission lines, presumably because they consist of pair plasma.

A multi-wavelength observation campaign is desirable for the comprehension of SS 433, because the behavior and relation of the system components, a synchrotron jet, an optically-thick accretion disk, and a high-energy jet engine, can be studied only with a multi-wavelength campaign $[10,3,4,12]$. Especially, radio monitoring to diagnose the state of the system, optical spectroscopy to determine the precessional phase, and X-ray observation of the core of the system are essential. We present preliminary results from an intensive multi-wavelength observation campaign of the source in April 2006 with the X-ray observatory Suzaku and several large radio and optical telescopes.

\section{Observations}

The source has been observed with the X-ray astronomical satellite Suzaku [16] at MJD = 53830 and 53833 (Table 1). The orbital phases at the observations are 1.0 and 0.22 , respectively [7]. An optical-IR-radio observation campaign was performed to cover the periods of the Suzaku observations (Table 2-4). Spectra have been taken with the 6-m Telescope (BTA) at the Special Astrophysical Observatory RAS (SAO RAS), the 122-cm Telescope at the Padova-Asiago Observatory, the 150-cm Telescope at the Gunma Astronomical Observatory [17], and the Nayuta Telescope at the Nishi-Harima Astronomical Observatory. Photometric data have been obtained with the KGB-38 Telescope at the Crimean Astrophysical Observatory, a 40-cm Telescope at the Kyoto University, MITSuME Akeno $50 \mathrm{~cm}$ [11] at the Akeno Observatory, MITSuME OAO 50 $\mathrm{cm}[11]$ at the Okayama Astrophysical Observatory (OAO), the 51-cm Telescope at the Osaka Kyoiku University [20], and telescopes in VSNET [9] and in the Variable Star Observers League in Japan (VSOLJ). Infrared photometry data have been obtained with the IRSF 1.4-m Telescope [8] at the South African Astronomical Observatory (SAAO). The radio activity from $1.0 \mathrm{GHz}$ to 21.7 $\mathrm{GHz}$ has been monitored with the RATAN-600 at SAO in the period covering the campaign. The 32-m radio telescope (RTF-32) at the Institute of Applied Astronomy RAS (IAA RAS) and the Nobeyama Millimeter Array at the Nobeyama Radio Observatory have also participated in the campaign.

\section{Precessional Phase}

The Doppler shifts of the jets of SS 433 measured with the X-ray Imaging Spectrometers (XIS) [13] on Suzaku and the optical spectrometers are shown in Fig. 1. This plot provides information on the geometrical configuration of the system at that time. Observed Doppler shifts deviate from the sinusoidal curves of the five-parameter kinematic model [15] due to the nodding motion [6]. From Fig. 1, it is confirmed that the campaign has been performed at a precessional phase when the inclination angle of the jet axis slightly exceeds $90^{\circ}$. 


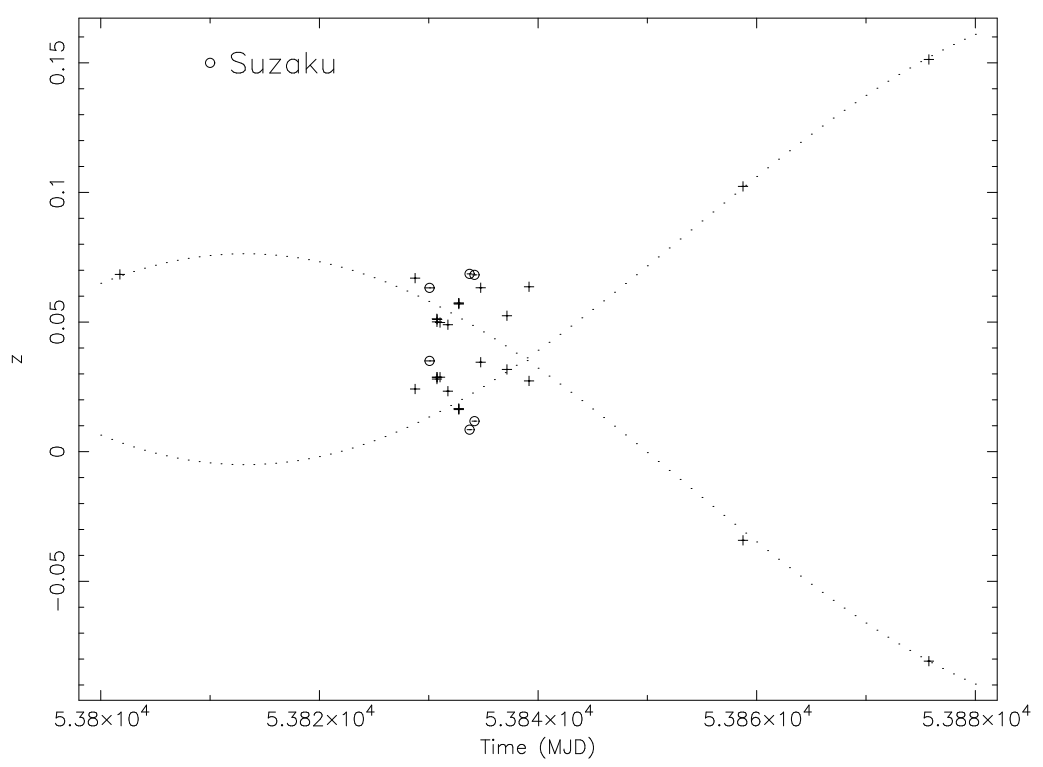

Figure 1: Doppler shifts. Optical data (crosses) and X-ray data (open circles) are plotted. Dotted lines are sinusoidal curves [15] shifted to fit the observational points.

\section{Light Curves}

Radio monitoring of the activity of the source is essential to a multi-wavelength campaign [19]. The radio light curves during the campaign are shown in Fig. 2. The curves exhibit five radio flares at $\mathrm{MJD}=53837,53847,53855,53866$, and 53870, suggesting that the source has been in the active state successively ejecting massive jets. Optical spectroscopic observations with the $122-\mathrm{cm}$ Telescope/Padova-Asiago and Nayuta/Nishi-Harima coincide with the flare at MJD $=53837$. It is interesting that no lines are detected from the coinciding observation of Nayuta. The observations with Suzaku have been performed before the first detected flare. At that time, the $2 \mathrm{GHz}$ flux densities fluctuate around $1 \mathrm{Jy}$, indicating a high activity even before the first detected flare. Suzaku might have observed the source in the interval between two flares.

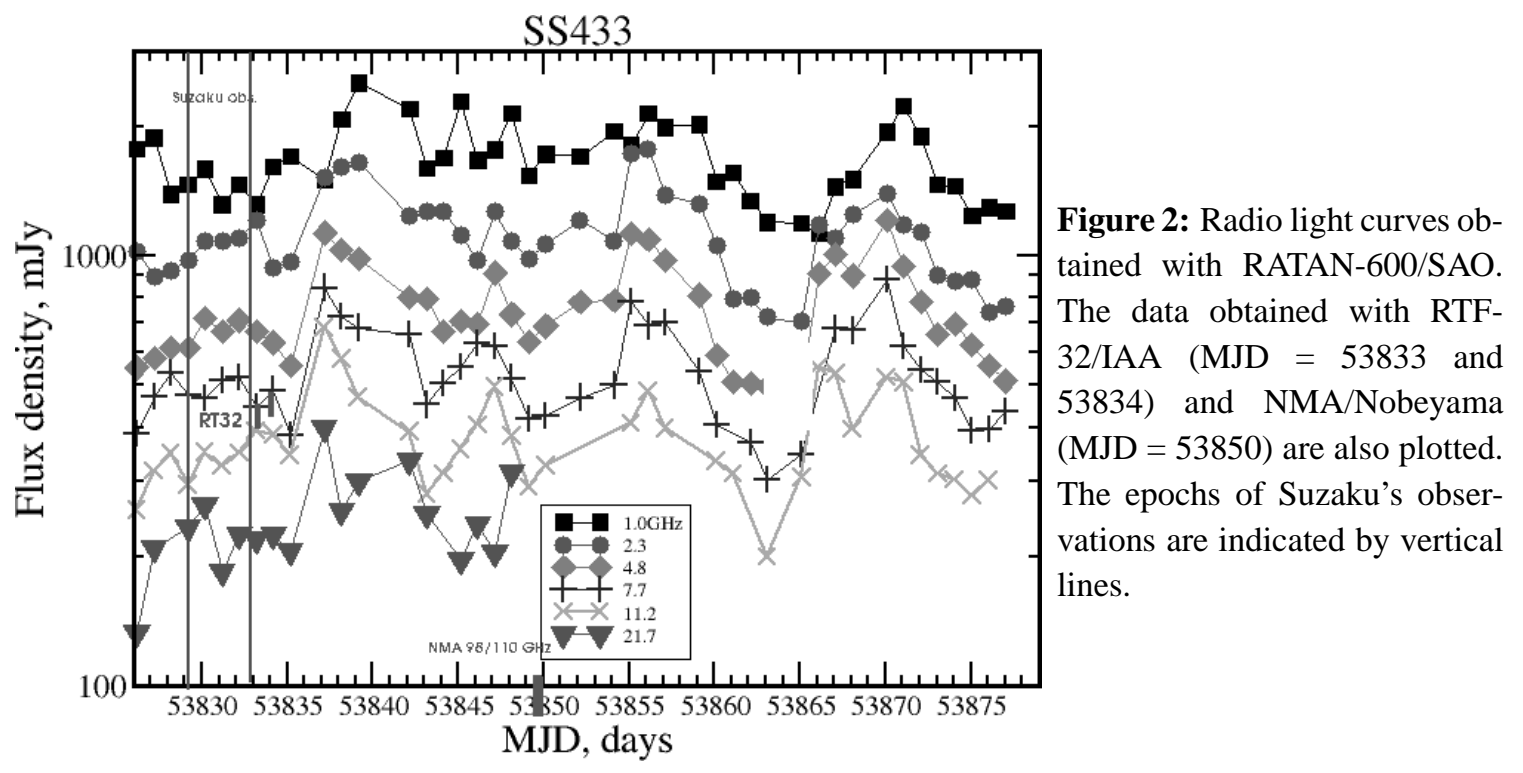

The VRI optical light curves are shown in Fig. 3. The participating observatories/organizations 
are listed in Table 3. A continuous observation with MITSuME OAO $50 \mathrm{~cm}$ coincides with the Suzaku observation out of eclipse.

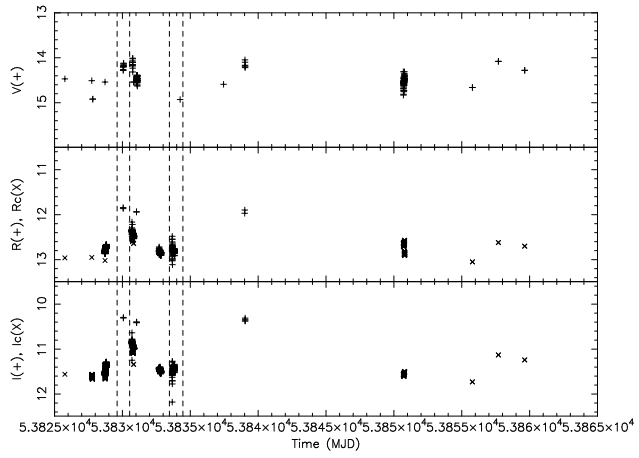

Figure 3: Optical light curves. The periods of two Suzaku observations are indicated by vertical dashed lines.

The X-ray light curves are shown in Fig. 4. The first observation has been performed during an eclipse. The X-ray flux recovers from the minimum, which is slightly different from the prediction by [7]. The cause of the shift is not known yet. In the second observation, the flux shows a significant variability, especially in the hard band above $5 \mathrm{keV}$.

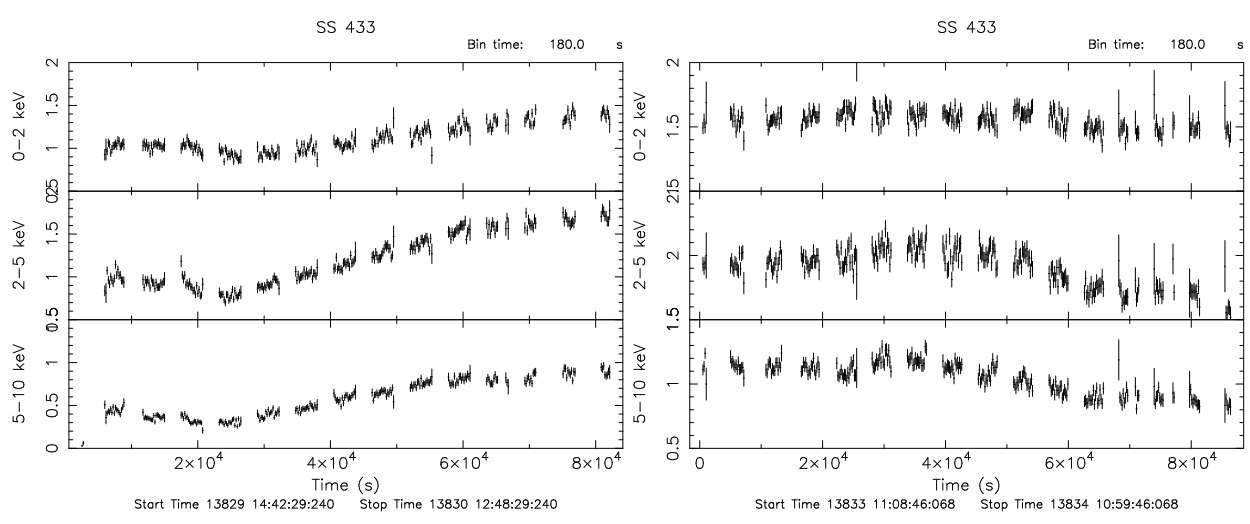

Figure 4: X-ray light curves obtained with XIS/Suzaku. Left: 2006/04/04 (MJD = 53829). Right: 2006/04/08 (MJD = 53833).

An example of simultaneous X-ray/optical observation is shown in Fig. 5. Correlation between these bands is to be searched. The optical emission from a geometrically thick super-critical accretion disk may precede to the X-ray emission from the hot base of the jet, while the emission from the downstream optical jet will lag behind the X-ray.

\section{Radio spectra}

Several radio spectra are plotted in Fig. 6. The sampled dates are MJD $=53829.192$ (coinciding with Suzaku's observation in eclipse), 53833.181 (coinciding with Suzaku's observation out of eclipse), 53837.170 (a flare peak), and 53850 (with NMA data up to $110.21 \mathrm{GHz}$ ). The spectrum at the flare peak is flatter, which is a characteristics of optically thick synchrotron sources such as small expanding jet plasma. Other spectra are consistent with that of optically thin synchrotron emission with a spectral index of $\sim 0.6$. It should be noted that the power-law like spectrum is extended up to $110.21 \mathrm{GHz}$. And even the flux densities other than the flare peak are higher than 


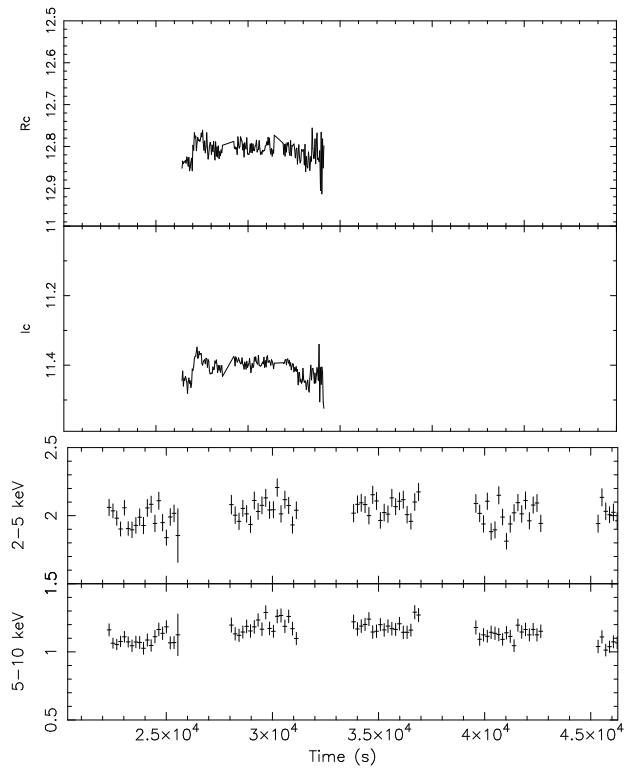

Figure 5: Simultaneous X-ray and optical observations. From top to bottom: Rc magnitude obtained with MITSuME OAO $50 \mathrm{~cm}$, Ic with MITSuME OAO $50 \mathrm{~cm}, 2-5 \mathrm{keV}$ count rate obtained with XIS/Suzaku, and 5-10 keV count rate with $\mathrm{XIS} /$ Suzaku. The horizontal range is from MJD $=53833.7$ to 53834.0 .

previously observed values (see, e.g., Fig. 3 in [18]). It also supports the assumption that the source has been in the active state during the campaign.

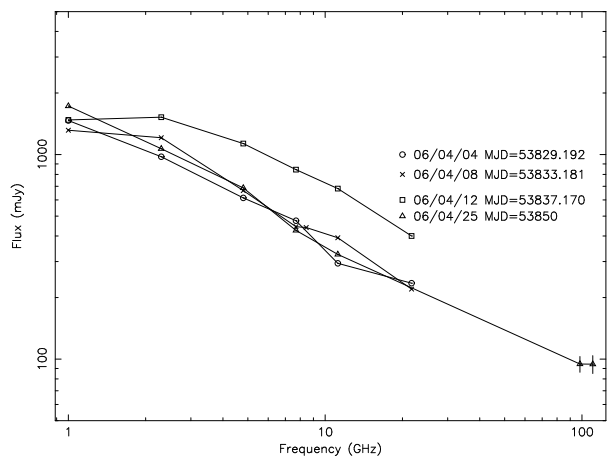

Figure 6: Radio spectra sampled at MJD = 53829.192, 53833.181, 53837.170, and 53850.

\section{Multi-wavelength Spectrum}

An exactly simultaneous multi-wavelength spectrum taken at MJD $=53833$ is plotted in Fig. 7 . Hard X-ray data of HXD/Suzaku and IR data of IRSF are not yet reducted. A spectral model for each energy band is plotted: The radio flux densities are expressed with a power-law model attenuated by interstellar matter of $A_{\mathrm{v}}=8$ or $N_{\mathrm{H}}=1.57 \times 10^{22} \mathrm{~cm}^{-2}$. The optical model is the sum of a multicolor disk model with $T_{\text {in }}=10^{5} \mathrm{~K}$ and a blackbody emission from a companion star with $T=1.5 \times 10^{4} \mathrm{~K}$, both of which are attenuated by interstellar matter of $A_{\mathrm{v}}=8$. The X-ray model consists of bremsstrahlung continuum and emission lines attenuated by $N_{\mathrm{H}}=1.3 \times 10^{23} \mathrm{~cm}^{-2}$.

\section{Summary}

A radio-IR-optical-X-ray observation campaign has been performed in April 2006, when the inclination of the jet axis slightly exceeds $90^{\circ}$. SS 433 has been active in the radio band and 


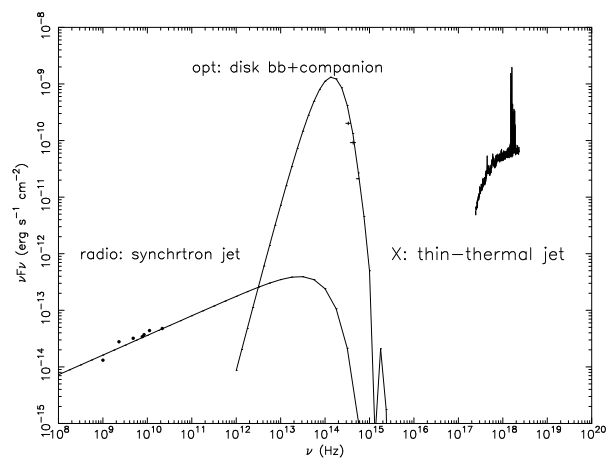

Figure 7: Exactly simultaneous multiwavelength spectrum taken at MJD $=53833$. Radio: RATAN-600 and RTF-32. Optical: VSNET and MITSUME OAO $50 \mathrm{~cm}$. X-ray: XIS/Suzaku. A spectral model for each energy band is plotted in solid line.

exhibited five massive jet ejection events during the campaign. One of the ejection events coincides with optical spectroscopic observations. The source in and out of eclipse has been observed with the X-ray astronomical satellite Suzaku. In the data out of eclipse, the X-ray flux shows variation of a factor of 2 in a day, suggesting that the source are in the active state like that observed in 1979 with Einstein $[18,2]$. The exactly simultaneous multi-wavelength spectrum can be expressed with a combination of three component, a synchrotron power-law model in the radio band, a multi-color blackbody model in the optical band, and a thin-thermal plasma model in the X-ray band. Temporal and spectral investigation are in progress.

\section{Acknowledgments}

TK is supported by a 21st Century COE Program at Tokyo Tech "Nanometer-Scale Quantum Physics" by the Ministry of Education, Culture, Sports, Science and Technology. This work is supported by the Japan-Russia Research Cooperative Program of Japan Society for the Promotion of Science. The studies are supported by the Russian Foundation Base Research (RFBR) grant N 05-02-17556 and the mutual RFBR and Japan Society for the Promotion of Science (JSPS) grant N 05-02-19710.

\begin{tabular}{|c|c|c|c|c|c|}
\hline \multicolumn{2}{|c|}{ Start } & \multicolumn{2}{|c|}{ End } & $\begin{array}{c}\text { Expos. } \\
(\mathrm{ks})\end{array}$ & Remark \\
\hline \multicolumn{5}{|c|}{ OObservatory: Suzaku [16]. PI: N. Kawai. } & \multirow{3}{*}{ Med Eclipse. } \\
\hline 2006/04/04 14:40 & $(53829.6108)$ & 2006/04/05 12:47 & $(53830.5326)$ & 40 & \\
\hline 2006/04/08 11:04 & $(53833.4610)$ & 2006/04/09 10:59 & $(53834.4578)$ & 40 & \\
\hline
\end{tabular}

Table 1: X-Ray observation $\log$.

\section{References}

[1] D. L. Band, and J. E. Grindlay, Synchrotron and inverse Compton emission from expanding sources in jets - Application to SS 433, ApJ 311 (1986) 595.

[2] D. L. Band, Comparison of Einstein and EXOSAT X-ray observations of SS 433, ApJ 336 (1989) 937.

[3] S. K. Chakrabarti, B. G. Anandarao, S. Pal, S. Mondal, A. Nandi, A. Bhattacharyya, S. Mandal, R. Sagar, et al., SS 433: results of a recent multiwavelength campaign, MNRAS 362 (2005) 957. 


\begin{tabular}{|c|c|c|c|}
\hline Start & $(\mathrm{MJD})$ & \multicolumn{2}{|c|}{$\begin{array}{l}\text { Exposure } \\
\text { (s) }\end{array}$} \\
\hline \multicolumn{4}{|c|}{ Telescope: BTA. Observatory: SAO RAS. PI: S. Fabrika. } \\
\hline \multicolumn{4}{|c|}{ Telescope: $122 \mathrm{~cm}$. Observatory: Padova-Asiago. PI: T. Iijima. } \\
\hline 2006/04/12 03:12:57 & $(53837.1340)$ & 1200 & \\
\hline 2006/04/14 02:37:01 & $(53839.1090)$ & 1200 & \\
\hline \multicolumn{4}{|c|}{ Telescope: $150 \mathrm{~cm}$ [17]. Observatory: Gunma. PI: K. Kinugasa. } \\
\hline 2006/03/07 18:00 & $(53801.75)$ & 120 & \\
\hline 2006/04/02 18:00 & $(53827.75)$ & 180 & Line not detected. \\
\hline 2006/04/05 18:00 & $(53830.75)$ & 120 & \\
\hline 2006/04/06 18:00 & $(53831.75)$ & 300 & \\
\hline 2006/04/07 18:00 & $(53832.75)$ & 300 & \\
\hline 2006/04/09 18:00 & $(53834.75)$ & 300 & \\
\hline 2006/05/03 18:00 & $(53858.75)$ & 180 & \\
\hline 2006/05/20 18:00 & $(53875.75)$ & 180 & \\
\hline \multicolumn{4}{|c|}{ Telescope: Nayuta. Observatory: Nishi-Harima. PI: S. Ozaki. } \\
\hline 2006/04/03 18:04:52 & $(53828.7534)$ & 1800 & \\
\hline 2006/04/05 17:55:00 & $(53830.7465)$ & $1800 \times 2$ & \\
\hline 2006/04/07 17:48:03 & $(53832.7417)$ & 592,540 & \\
\hline 2006/04/12 08:07:16 & $(53837.3384)$ & 1800 & Line not detected. \\
\hline
\end{tabular}

Table 2: Spectroscopic observation log.

[4] A. M. Cherepashchuk, R. A. Sunyaev, S. N. Fabrika, K. A. Postnov, S. V. Molkov, E. A. Barsukova, E. A. Antokhina, T. R. Irsmambetova, et al., INTEGRAL observations of SS433: Results of a coordinated campaign, A\&A 437 (2005) 561.

[5] S. Fabrika, The jets and supercritical accretion disk in SS433, Astrophysics and Space Physics Reviews 12 (2004) 1.

[6] Gies, D. R., M. V. McSwain, R. L. Riddle, Z. Wang, P. J. Wiita, and D. W. Wingert, The Spectral Components of SS 433, ApJ 566 (2002) 1069.

[7] S. A. Gladyshev, V. P. Goranskii, A. M. Cherepashchuk, Photometric Investigations of SS 433-Results of 1979-1986 Observations, Sov. Astron. 31 (1987) 541.

[8] R. Kandori, N. Kusakabe, M. Tamura, Y. Nakajima, T. Nagayama, C. Nagashima, J. Hashimoto, J. Hough, et al., SIRPOL: a JHK $\mathrm{S}_{\mathrm{s}}$-simultaneous imaging polarimeter for the IRSF 1.4-m telescope, Proc. SPIE 6269 (2006).

[9] T. Kato, M. Uemura, R. Ishioka, D. Nogami, C. Kunjaya, H. Baba, and H. Yamaoka, Variable Star Network: World Center for Transient Object Astronomy and Variable Stars, PASJ 56 (2004) 1.

[10] T. Kotani, D. Band, A. M. Cherepashchuk, R. M. Hjellming, N. Kawai, M. Matsuoka, M. Namiki, T. Oka, et al., Multi-wavelength observations of the jet sources SS 433 and XTE J1748-288, Astronomische Nachrichten 320 (1999) 335.

[11] T. Kotani, N. Kawai, K. Yanagisawa, J. Watanabe, M. Arimoto, H. Fukushima, T. Hattori, M. Inata, et al., MITSuME-Multicolor Imaging Telescopes for Survey and Monstrous Explosions, in proceedings of 4th Workshop on Gamma-Ray Burst in the Afterglow Era, (2005) 755.

[12] T. Kotani, S. A. Trushkin, R. Valiullin, K. Kinugasa, S. Safi-Harb, N. Kawai, and M. Namiki, $A$ Massive Jet Ejection Event from the Microquasar SS 433 Accompanying Rapid X-Ray Variability, ApJ 637 (2006) 486. 


\begin{tabular}{|c|c|c|c|}
\hline $\begin{array}{c}\text { Start } \\
(\mathrm{MJD})\end{array}$ & $\begin{array}{c}\text { End } \\
(\mathrm{MJD})\end{array}$ & Frames & Remark \\
\hline \multicolumn{4}{|c|}{ Instrument: KGB-38. Observatory: Crimean. PI:T. Irsmambetova. } \\
\hline 53830.05325 & 53830.08605 & $\mathrm{~V}: 10, \mathrm{R}: 2, \mathrm{I}: 2$ & \\
\hline 53831.03587 & 53831.10694 & $\mathrm{~V}: 45, \mathrm{R}: 2, \mathrm{I}: 2$ & \\
\hline 53839.00955 & 53839.04312 & V: $6, \mathrm{R}: 2, \mathrm{I}: 2$ & \\
\hline \multicolumn{4}{|c|}{ Telescope: 40 cm. Observatory: Kyoto U. PI: K. Kubota. } \\
\hline 53826.72946 & 53826.83986 & C: 243 & \\
\hline 53828.72057 & 53828.79068 & C: 91 & \\
\hline 53841.67304 & 53841.82313 & C: 316 & \\
\hline 53848.74419 & 53848.81521 & C: 171 & \\
\hline 53850.79558 & 53850.81689 & C: 55 & \\
\hline 53852.80954 & 53852.82616 & C: 51 & \\
\hline 53886.72074 & 53887.24026 & C: 51 & \\
\hline \multicolumn{4}{|c|}{ Telescope: MITSuME Akeno $50 \mathrm{~cm}$ [11]. Observatory: Akeno. PI: T. Shimokawabe } \\
\hline 53827.76578 & 53827.80385 & V: $47, \mathrm{I}: 47$ & \\
\hline 53828.72676 & 53828.79979 & V: 91, I: 91 & \\
\hline 53830.73239 & 53830.80104 & V: 90, I: 90 & \\
\hline \multicolumn{4}{|c|}{ Telescope: MITSuME OAO $50 \mathrm{~cm}$ [11]. Observatory: OAO. PI: S. Yanagisawa. } \\
\hline 53828.68783 & 53828.83571 & g': 321, Rc: 321 , Ic: 321 & \\
\hline 53830.69849 & 53830.84740 & g': 360, Rc: 360, Ic: 360 & \\
\hline 53832.70071 & 53832.84003 & g': 340, Rc: 340, Ic: 340 & \\
\hline 53833.67184 & 53833.84119 & g': 220, Rc: 220, Ic: 220 & \\
\hline \multicolumn{4}{|c|}{ Telescope: 51 cm [20]. Observatory: Osaka Kyoiku U. PI: S. Nishiyama. } \\
\hline 53825.78 & 53825.80 & V: $1, \mathrm{R}: 1, \mathrm{I}: 1$ & \\
\hline 53828.77 & 53828.79 & $\mathrm{~V}: 1, \mathrm{R}: 1, \mathrm{I}: 1$ & \\
\hline 53849.73734 & 53849.77499 & $\mathrm{~V}: 1, \mathrm{R}: 15, \mathrm{I}: 1$ & \\
\hline 53850.64105 & 53850.76927 & V: 1, R: 30, I: 2 & \\
\hline 53855.72479 & 53855.75851 & R: 38 & \\
\hline 53857.76686 & 53857.78237 & $\mathrm{R}: 14$ & \\
\hline 53858.68045 & 53858.68667 & $\mathrm{R}: 10$ & \\
\hline 53859.69379 & 53859.20651 & R: 19 & \\
\hline \multicolumn{4}{|c|}{ Organization: VSNET [9]. } \\
\hline 53823.79792 & 53823.80069 & V: 1, Rc: 1 , Ic: 1 & S. Kiyota (Ibaraki, Japan) \\
\hline 53823.81339 & - & $\mathrm{V}: 1$ & H. Maehara (Saitama, Japan) \\
\hline 53824.78819 & 53823.83194 & V: 1, Rc: 1 , Ic: 1 & S. Kiyota (Ibaraki, Japan) \\
\hline 53827.75850 & 53827.76653 & V: 1, Rc: 1, Ic: 1 & K. Nakajima (Mie, Japan) \\
\hline 53827.81163 & - & $\mathrm{V}: 1$ & H. Maehara (Saitama, Japan) \\
\hline 53828.73766 & 53828.79404 & V: 1, Rc: 1, Ic: 1 & K. Nakajima (Mie, Japan) \\
\hline 53830.81929 & 53830.82405 & V: 1, Rc: 1, Ic: 1 & H. Maehara (Saitama, Japan) \\
\hline 53834.24931 & - & $\mathrm{V}: 1$ & D. J. Mendicini (Spain) \\
\hline 53837.44860 & 53837.44990 & $\mathrm{~B}: 1, \mathrm{~V}: 1$ & D. J. Mendicini (Spain) \\
\hline 53855.78171 & 53855.78499 & V: 1, Rc: 1 , Ic: 1 & H. Maehara (Saitama, Japan) \\
\hline 53857.70069 & 53857.70138 & V: 1, Rc: 1, Ic: 1 & S. Kiyota (Ibaraki, Japan) \\
\hline 53859.64028 & 53859.64097 & V: 1, Rc: 1 , Ic: 1 & S. Kiyota (Ibaraki, Japan) \\
\hline 53886.64912 & 53886.65486 & V: 1, Rc: 1, Ic: 1 & S. Kiyota (Ibaraki, Japan) \\
\hline
\end{tabular}

Table 3: Photometric observation $\log$. 


\begin{tabular}{ccll}
\hline \hline $\begin{array}{c}\text { Start } \\
(\text { MJD })\end{array}$ & $\begin{array}{c}\text { End } \\
(\text { MJD) }\end{array}$ & Frames & Remark \\
\hline \hline Organization: VSOLJ. & & \\
53824.80267 & 53824.82122 & C: 24 & K. Nakajima (Mie, Japan) \\
53825.75694 & 53825.75833 & V: 1, Rc: 1, Ic: 1 & S. Kiyota (Ibaraki,Japan) \\
53825.78413 & 53825.82137 & V: 1, Rc: 1, Ic: 1 & K. Nakajima (Mie, Japan) \\
53827.74097 & 53827.79264 & V: 1, Rc: 1, Ic: 98 & S. Kiyota (Ibaraki, Japan) \\
53828.71944 & 53828.81230 & V: 1, Rc: 1, Ic: 184 & S. Kiyota (Ibaraki, Japan) \\
53830.73455 & 53830.79178 & V: 11, Rc: 1, Ic: 143 & S. Kiyota (Ibaraki, Japan) \\
53842.14863 & 53842.17043 & Rc: 59 & H. Maehara (Saitama,Japan) \\
53850.66944 & 53850.78032 & Rc: 204 & K. Nakajima (Mie,Japan) \\
53850.69490 & 53850.79198 & V: 67, Rc: 65, Ic: 66 & S. Kiyota (Ibaraki,Japan) \\
\hline $\begin{array}{l}\text { Telescope: IRSF 1.4m [8]. Observatory: SAAO. PI: T. Nagata. } \\
53830.15679\end{array} 53830.15889$ & J: 3, H: 3, Ks: 3 & \\
\hline \hline
\end{tabular}

Table 3: Photometric observation log. (Cont'd)

\begin{tabular}{|c|c|c|c|c|}
\hline \multicolumn{2}{|l|}{ Start } & \multicolumn{2}{|c|}{ End } & \multirow[t]{2}{*}{ Remark } \\
\hline & $(\mathrm{MJD})$ & & (MJD) & \\
\hline \multicolumn{5}{|c|}{ T"Telescope: RATAN-600. Observatory: SAO RAS. PI: S. Trushkin. } \\
\hline 2006/04/01 & $(53826)$ & $2006 / 05 / 22$ & $(53877)$ & $1.0 \mathrm{GHz}-21.7 \mathrm{GHz}$ \\
\hline \multicolumn{5}{|c|}{ Telescope: RTF-32. Observatory: IAA RAS. PI: S. Trushkin. } \\
\hline 2006/04/08 01:06 & $(53833.0458)$ & 2006/04/08 08:52 & $(53833.3694)$ & 4 expos. $2.3 \mathrm{GHz} 8.45 \mathrm{GHz}$ \\
\hline 2006/04/09 00:53 & $(53834.0368)$ & 2006/04/09 08:50 & $(53834.3680)$ & 5 expos. \\
\hline \multicolumn{5}{|c|}{ Telescope: Nobeyama Millimeter Array. Observatory: Nobeyama. PI: K. Nakanishi } \\
\hline 2006/04/25 22:10 & $(53850.9236)$ & $2006 / 04 / 2522: 30$ & $(53850.9375)$ & $110.21 \mathrm{GHz}, 98.201 \mathrm{GHz}$ \\
\hline
\end{tabular}

Table 4: Radio observation $\log$.

[13] K. Koyama, H. Tsunemi, T. Dotani, M. W. Bautz, K. Hayashida, T. Tsuru, H. Matsumoto, Y. Ogawara, et al., X-Ray Imaging Spectrometers (XIS) on Board Suzaku, PASJ, (2006) in press.

[14] B. Margon, Observations of SS 433, ARA\&A 22 (1984). 507.

[15] B. Margon, S. F. Anderson, Ten years of SS 433 kinematics, ApJ 347 (1989) 448.

[16] K. Mitsuda, M. Bautz, H. Inoue, R. Kelley, K. Koyama, H. Kunieda, K. Makishima, Y. Ogawara, et al., The X-Ray Observatory Suzaku, PASJ, (2006) in press.

[17] H. Obayashi, O. Hashimoto, E. Nishihara, and K. Kinugasa, The advantages of Gunma Astronomical Observatory, in proceedings of Third Rome Workshop on Gamma-Ray Bursts in the Afterglow Era, (2004) 540 .

[18] E. R. Seaquist, W. S. Gilmore, K. J. Johnston, and J. E. Grindlay, Simultaneous radio and X-ray activity in SS 433, ApJ 260 (1982) 220.

[19] S. A. Trushkin, N. N. Bursov, and N. A. Nizhelskij, The multifrequency monitoring of microquasars. SS433, in Bulletin of the Special Astrophysical Observatory 56 (2003) 57 [astro-ph/ 0403037 ].

[20] T. Yokoo, J. Arimoto, K. Matsumoto, A. Takahashi, and K. Sadakane, VRI CCD photometric observations of SN 1994 in M51, PASJ 46 (1994) L191. 